\title{
Single-mode regime in large-mode-area rare-earth-doped rod-type PCFs
}

Poli, F.; Cucinotta, A.; Passaro, D.; Selleri, S.; Lægsgaard, Jesper; Broeng, Jes

Published in:

IEEE Journal of Selected Topics in Quantum Electronics

Link to article, DOI:

10.1109/JSTQE.2008.2010265

Publication date:

2009

Document Version

Publisher's PDF, also known as Version of record

Link back to DTU Orbit

Citation (APA):

Poli, F., Cucinotta, A., Passaro, D., Selleri, S., Lægsgaard, J., \& Broeng, J. (2009). Single-mode regime in largemode-area rare-earth-doped rod-type PCFs. IEEE Journal of Selected Topics in Quantum Electronics, 15(1), 5460. https://doi.org/10.1109/JSTQE.2008.2010265

\section{General rights}

Copyright and moral rights for the publications made accessible in the public portal are retained by the authors and/or other copyright owners and it is a condition of accessing publications that users recognise and abide by the legal requirements associated with these rights.

- Users may download and print one copy of any publication from the public portal for the purpose of private study or research.

- You may not further distribute the material or use it for any profit-making activity or commercial gain

- You may freely distribute the URL identifying the publication in the public portal 


\title{
Single-Mode Regime in Large-Mode-Area Rare-Earth-Doped Rod-Type PCFs
}

\author{
Federica Poli, Annamaria Cucinotta, Member, IEEE, Davide Passaro, Student Member, IEEE, \\ Stefano Selleri, Senior Member, IEEE, Jesper Lægsgaard, and Jes Broeng
}

\begin{abstract}
In this paper, large-mode-area, double-cladding, rare-earth-doped photonic crystal fibers are investigated in order to understand how the refractive index distribution and the mode competition given by the amplification can assure singlemode propagation. Fibers with different core diameters, i.e., 35, 60 , and $100 \mu \mathrm{m}$, are considered. The analysis of the mode effective index, overlap, effective area, gain, and power evolution along the doped fiber provides clear guidelines on the fiber physical characteristics to be matched in the fabrication process to obtain a truly or effectively single-mode output beam.
\end{abstract}

Index Terms-Photonic crystal fibers (PCFs), rare-earth-doped fibers, rod-type fibers, single-mode regime.

\section{INTRODUCTION}

$\mathbf{H}$ IGH- and medium-power applications of fiber lasers are gaining interest day by day in many different fields ranging from spectroscopy to medicine, from monitoring and sensing to micromachining and material processing [1]. The wellestablished fiber and doped fiber technology is one of the keys to this success. In particular, rare-earth-doped double-cladding (DC) fibers [2], made as step-index fibers with a polymer outer cladding, are usually exploited for these kinds of applications. Recently, the advent of photonic crystal fibers (PCFs) with their novel optical properties [3] has further boosted the process. Large-mode area (LMA) DC PCFs, usually called air-clad fibers, seem to be particularly promising [4]-[6]. In these fibers, the LMA structure, with a doped core in a photonic crystal inner cladding, is placed inside an air-clad pump guide, characterized by a ring of large air holes. A very high numerical aperture is provided for the pump core/outer cladding, thus allowing efficient coupling of light from inexpensive high-power pump diodes having a poor beam quality. The main advantage of the PCF technology is related to the possibility, due to the high index control, to fabricate LMA single-mode doped cores, which allow to reach high power levels, avoiding nonlinear effects and providing a good overlap between pump and signal. Moreover, since air holes are used instead of polymers for the air-clad PCF

Manuscript received September 1, 2008. Current version published February 4, 2009.

F. Poli, A. Cucinotta, D. Passaro, and S. Selleri are with the University of Parma, Parma 43100, Italy (e-mail: stefano.selleri@unipr.it).

J. Lægsgaard is with the Department of Photonics Engineering, Technical University of Denmark, DK-2800 Kgs. Copenhagen, Denmark (e-mail: jl@com.dtu.dk).

J. Broeng is with the Crystal Fibre A/S, 3460 Birkerod, Denmark (e-mail: jb@ crystal-fibre.com).

Digital Object Identifier 10.1109/JSTQE.2008.2010265 outer cladding [6], the thermal conductivity is greatly improved compared to the traditional DC fibers, and the material degradation is avoided [7]. A recent development is the manufacture of DC PCFs, called rod-type fibers, with the outer dimension of a rod laser, i.e., a diameter of few millimeters [8]. While the large outer diameter provides a reduction of macro- and microbend losses, the device retains the stability and the performances of a fiber laser.

An important issue for these LMA PCFs is still to keep the excellent quality of the output beam, first of all by proper fiber designs that can assure the single-mode propagation. In fact, even if single-mode lasing has been experimentally demonstrated for Yb-doped rod-type fibers [8], [9], numerical simulations have previously shown that the single-mode regime of all-silica PCFs is significantly reduced when a core region as large as in rod-type fibers is considered [10]. This paper aims to provide guidelines on geometry and refractive index distribution in order to allow manufacturers to fabricate LMA DC doped PCFs that are single-mode, or effectively single mode at the lasing wavelength of interest, strongly related to the rare-earth element added in the fiber core.

The analysis will first focus on the modal properties of the fundamental and higher order modes as a function of the doped core refractive index. Its value, in fact, increases with the doping, and manufacturers often attempt to match again the pure silica one by counter doping [9]. Slight variations, above or below the value for silica used in the cladding region, can considerably change the guidance characteristics of the modes, in particular of the higher order ones. A down-doped core surrounded by a photonic crystal cladding, for example, can guide due to the microstructure [9] and even lead to a short-wavelength cutoff for the fundamental mode [11]. By means of the finite-element method (FEM), we will, in this paper, analyze fibers with three different values of the doped core diameter, i.e., 35, 60, and $100 \mu \mathrm{m}$, showing, in particular, the mode effective index and the mode field distribution-doped region overlap versus the core refractive index.

As a second step, the analysis will consider the propagation of the modes along the doped fiber, showing how their gain competition can lead to effectively single-mode propagation, even for fibers that from simpler analysis would appear multimode. Results of this analysis will further confirm conclusions obtained from the computed overlap about the mode field and doped material interaction. Population and propagation rate equations will be mainly applied to $\mathrm{Yb}$-doped PCFs, showing results at $1064 \mathrm{~nm}$. Extension to Er-doped fiber working at $1550 \mathrm{~nm}$ is straightforward. 


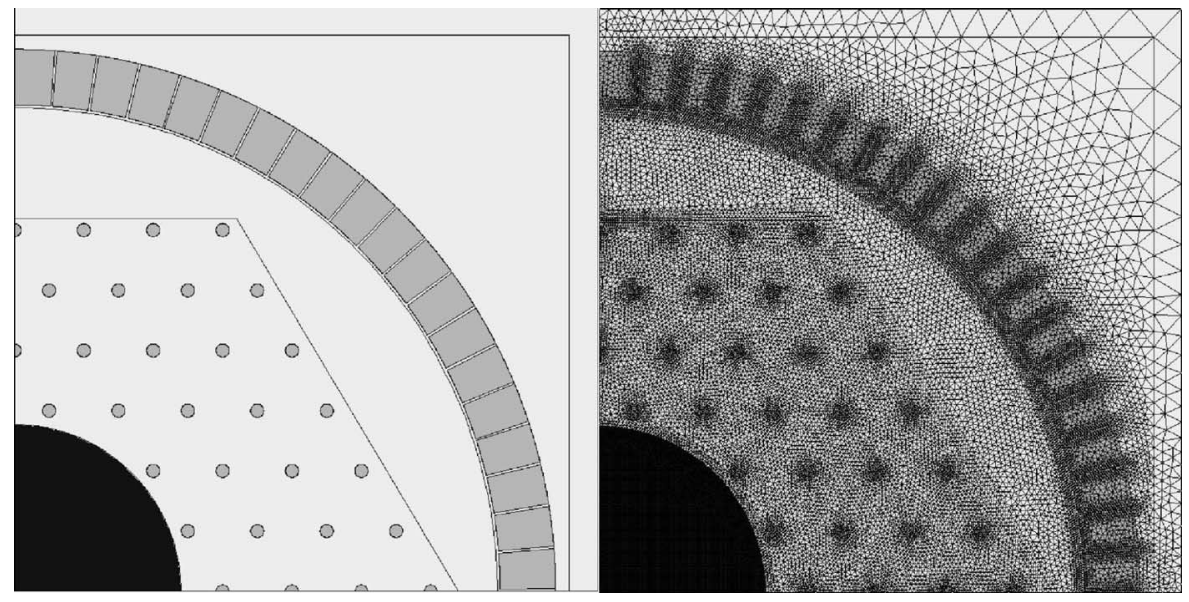

Fig. 1. One quarter of the LMA DC PCF under study (left) and the mesh used for the FEM computations (right). Light gray regions: pure silica; dark gray: air; black: doped core.

\section{NUMERICAL APPROACH}

\section{A. Modal Analysis}

The modal analysis is performed through a full-vector modal solver based on the FEM [12], [13], which requires as input data the actual refractive index profile of the doped fiber. The solver used here is based on the magnetic field curl-curl equation obtained by decoupling the Maxwell equations. Once the problem domain is suitably divided into a patchwork of finite subregions, called finite elements, the application of the standard variational FEM procedure yields the algebraic eigenproblem to be solved. When structural symmetries can be exploited, just one half or even one quarter of the whole domain can be considered. Fig. 1 shows the domain quarter used for the simulations. The vertical and horizontal boundaries, which cut the fiber core, define two possible symmetry axes where electric and magnetic perfect conductor conditions can be properly imposed to select all the possible fundamental mode (FM) and higher order mode (HOM) field distributions. The computed eigenvector represents the magnetic field distribution of the modes on the transverse fiber cross section, while the eigenvalue provides the attenuation constant and the effective index $n_{\text {eff }}$ of the modes. From the knowledge of the field, through the Poynting vector, the mode intensity, the effective area, and the mode overlap are straightforwardly computed.

\section{B. Propagation Analysis}

The propagation of the modes along the doped fiber is computed through a spatial model [14] that describes the power evolution by solving the propagation equations and the population rate equations, characterized, in terms of emission and absorption cross sections, dopant concentration, lifetime, etc., according to the considered doping material, i.e., ytterbium or erbium. Details of the model equations can be found in [15]. This spatial model uses the field distributions computed through the FEM to evaluate the intensities of the pump and of each propagating mode at the signal wavelength.
It is important to highlight that the model is not restricted to the description of a single mode, but it is able to consider, besides the pump, the propagation of more than one mode simultaneously, thus showing how they compete with each other in exploiting the pump energy and the inverted population. This model feature is very important to verify the effectively singlemode operation of the doped fibers.

\section{Mode Guiding Properties}

\section{A. 60- $\mu m$ Rod-Type PCF}

First, a detailed analysis of a LMA DC Yb-doped rod-type PCF with core diameter of $60 \mu \mathrm{m}$ is presented, showing later the fiber behavior by decreasing and increasing the core dimensions. The discussed PCF has a circular ytterbium-doped core, obtained by removing 19 air holes, surrounded by the triangular lattice of the inner cladding that is characterized by four air hole rings, $\Lambda=12.5 \mu \mathrm{m}$ and $d=2.375 \mu \mathrm{m}$, giving a ratio $d / \Lambda=0.19, \Lambda$ and $d$ being the pitch and air hole diameter, respectively [9]. The outer cladding is obtained through a compact ring of large air holes, separated by silica bridges about $400 \mathrm{~nm}$ wide and $10 \mu \mathrm{m}$ long. The refractive index of pure silica is equal to 1.45 , whereas for the doped core, different refractive index values are considered, ranging from 1.4495 to 1.45 , in order to investigate the effect of down-doping, which decreases the core index below the silica one. Fig. 1(left) shows a quarter of the studied structure, while Fig. 1(right) reports the mesh used for the FEM computations. The light gray region indicates the pure silica, the dark gray ones the air holes, and the black one the doped core.

The guiding properties of the FM and the first HOMs as a function of the doped core refractive index value have been evaluated. Fig. 2 reports the guided-mode effective index $n_{\text {eff }}$, computed at $1064 \mathrm{~nm}$. The effective index of the inner cladding space-filling mode (SFM) $n_{\mathrm{SFM}}$, which is assumed as the limit for the mode guidance, is reported in Fig. 2 as reference, but the present analysis does not depend on $n_{\mathrm{SFM}}$. The fiber is clearly multimode for a core index equal to the pure silica one. 


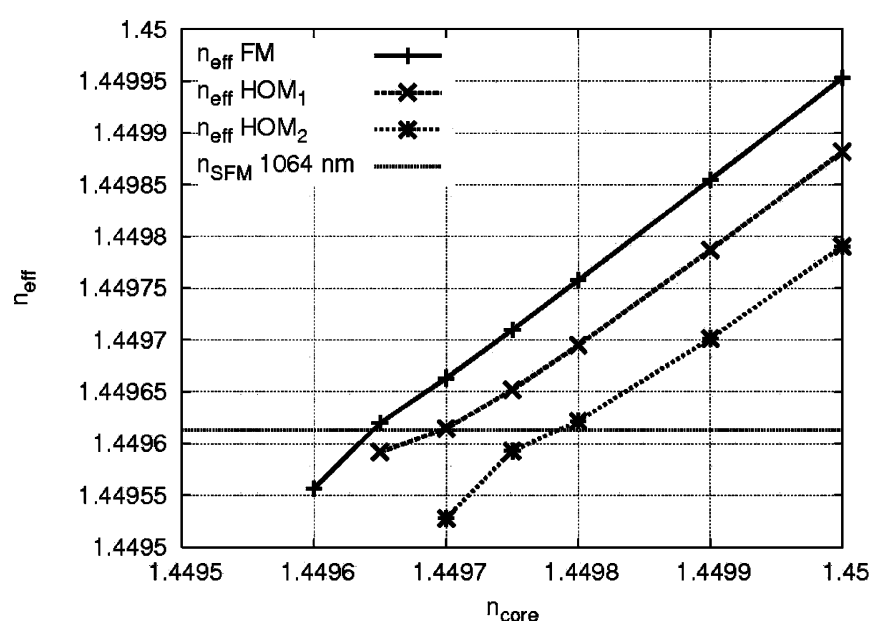

Fig. 2. Effective index of the FM and the first HOMs at $1064 \mathrm{~nm}$ versus the doped core refractive index. The value of the space-filling-mode effective index is reported as well.

However, as the core refractive index decreases, the guided mode $n_{\text {eff }}$ values become lower, down to the extent of crossing the SFM line. The modes thus become less and less confined, and eventually no longer guided as their effective index is lower than $n_{\mathrm{SFM}}$. In particular, this happens first for the HOMs, and then also for the FM, so that the fiber can be designed to be truly single-mode in a restricted range of the core refractive index, through a very precise choice of its value. Notice that when $n_{\text {core }}$ gets close to $n_{\mathrm{SFM}}$, the guided mode can be regarded as one of the many cladding modes, among these the one with the higher effective index has been selected, and its value has been reported in Fig. 2.

In practice, however, the refractive index is controlled to a certainly accuracy (typically around $\pm 1 \times 10^{-4}$ ), which makes it difficult to assure single-mode propagation through this technique. This point will be further discussed in the following sections when studying amplification.

The mode behavior just described when decreasing the core refractive index can also be observed in the magnetic field distribution of the FM, reported in Fig. 3. The mode field clearly spreads from the inner region of the core, displacing more and more into the microstructured cladding. The figure reports the computed solutions for $n_{\text {core }}=1.45,1.44975,1.44965$, and 1.4496. Similar behaviors have also been observed for the HOMs, of course considering higher values of the core refractive index.

It is also important to highlight how the outer ring of large air holes make up a strong barrier against the field leakage in the outer silica region of the rod-type PCF. For this reason, these fibers, even bent, suffer negligible leakage losses. Bending, however, may lead to undesired coupling between the core and inner-cladding modes.

Further insight is gained from the analysis of the guided-mode field overlap with the doped-core region and of the effective area $A_{\text {eff }}$, reported, respectively, in Figs. 4 and 5 versus $n_{\text {core }}$, still at $1064 \mathrm{~nm}$. By decreasing the core refractive index, the HOM overlap is reduced much more than the FM one, being, for ex- ample, with $n_{\text {core }}=1.4497$, only 0.64 and 0.21 , respectively, for $\mathrm{HOM}_{1}$ and $\mathrm{HOM}_{2}$, against 0.9 for the FM. The overlap is a very important parameter when considering the field propagation in a doped and pumped fiber. The values reported in Fig. 4 predict high differential gain to the advantage of the FM, so that the HOMs may be effectively suppressed even if their $n_{\text {eff }}$ is above $n_{\mathrm{SFM}}$.

The effective area still keeps very high values, around thousands of square micrometers, according to the achieved $n_{\text {core }}$. Of course, as the FM effective index becomes lower than $n_{\mathrm{SFM}}$ with decreasing $n_{\text {core }}$, the field spreads into the cladding providing very high effective area values that, however, do not correspond to a guided solution.

Finally, it is interesting to note that a strong reduction of $n_{\text {core }}$ gives the possibility to achieve the FM cutoff also as its effective index curve crosses the SFM one. This FM property can be exploited, for example, to suppress the amplified spontaneous emission in order to obtain amplification in the long wavelength range, for instance, in L band when considering an Er-doped rod-type PCF.

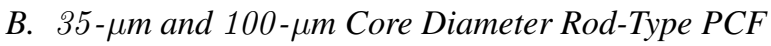

A similar analysis, in terms of effective index, field distribution, overlap, and effective area, has also been performed considering rod-type PCFs with different core diameters, i.e., 35 and $100 \mu \mathrm{m}$, whose geometrical characteristics are summarized in Table I. In particular, the first one has been already manufactured with a Yb-doped core, formed by removing seven air holes, a triangular lattice of the inner cladding characterized by $d / \Lambda=0.33$ with $\Lambda=12 \mu \mathrm{m}$, and an hexagonal air-cladding [8], whereas the second one has been designed by scaling the 60 $\mu \mathrm{m}$ PCF previously studied, and fabricated only in a passive version [9]. The values of $d$ and $\Lambda$ have been scaled by a factor 1.666, whereas the ratio $d / \Lambda$ has been unchanged, and the study has been performed at $1064 \mathrm{~nm}$.

The behavior of the two fibers qualitatively retraces the one already presented and, for the sake of brevity, it will not be discussed again. However, to highlight the differences arisen in the numerical results, it is very interesting to compare the overlap values of all the fibers, since this is an important figure of merit for the following discussion. Notice that the $35-\mu \mathrm{m}$ fiber air-cladding is hexagonal [8], unlike the circular one of the two other rod-type PCFs considered here. The overlap with the doped core of the FM and of the first HOM at $1064 \mathrm{~nm}$ is shown in Fig. 6(a) for the $35 \mu \mathrm{m}$ core PCF, and in Fig. 6(b) for the 60 and $100 \mu \mathrm{m}$ core diameter. For each fiber, the figures report a set of three curves always decreasing by reducing the core refractive index, however, with quite different slopes. While the overlaps in the $35-\mu \mathrm{m}$ rod-type PCF change only slightly with $n_{\text {core }}$, in the $60 \mu \mathrm{m}$ one, already discussed, changes are much more pronounced, and finally, the $100 \mu \mathrm{m}$ fiber presents very steep curves with strong variations in a limited range of $n_{\text {core }}$. Targeting a very high overlap value for the FM in this PCF, for example, as close to 1.0 as possible, the core refractive index must be very close to the pure silica one, resulting in HOMs that also maintain high overlaps. This could yield a 


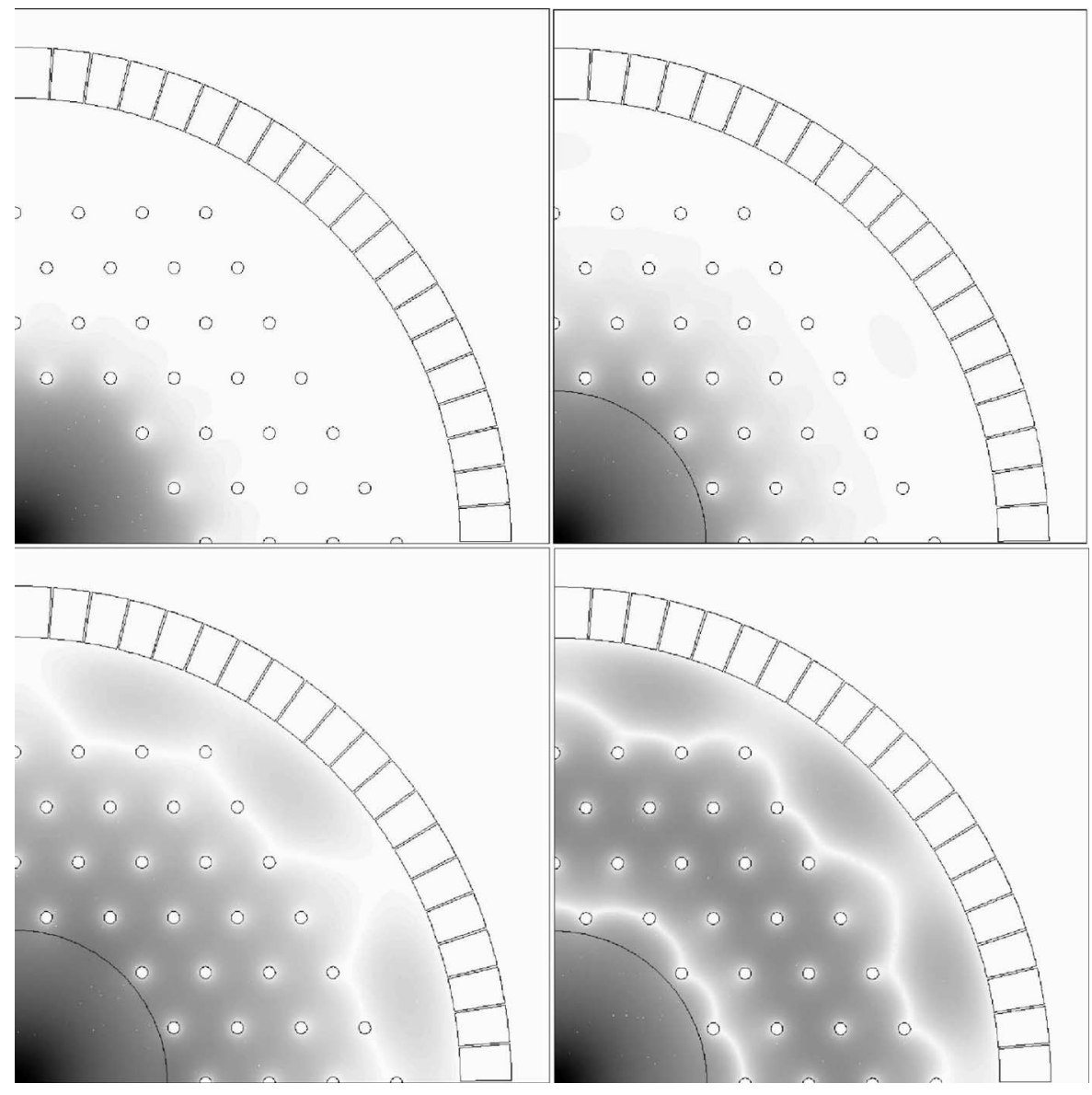

Fig. 3. Magnetic field distribution of the FM at $1064 \mathrm{~nm}$ with decreasing core refractive index. From left-top to right-bottom: $n_{\text {core }}=1.45,1.44975,1.44965$, and 1.4496 .

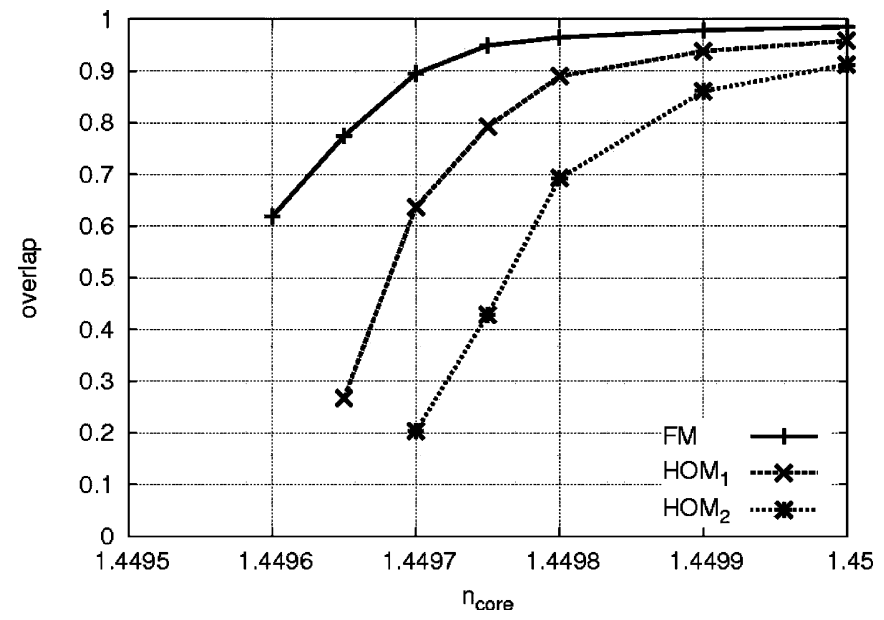

Fig. 4. Field overlap with the doped core of the FM and HOMs at $1064 \mathrm{~nm}$ versus the core refractive index.

strong competition between amplifying modes. On the contrary, by slightly reducing $n_{\text {core }}$, the HOMs are pushed close, or even below, the $n_{\mathrm{SFM}}$ curve and their overlap with the doped region decreases considerably.

For all the three fibers considered, a FM overlap around 0.9 assures $\mathrm{HOM}_{1}$ overlap values around 0.6 , and $\mathrm{HOM}_{2}$ ones below 0.3 , which are quite effective to actually guarantee a single-mode

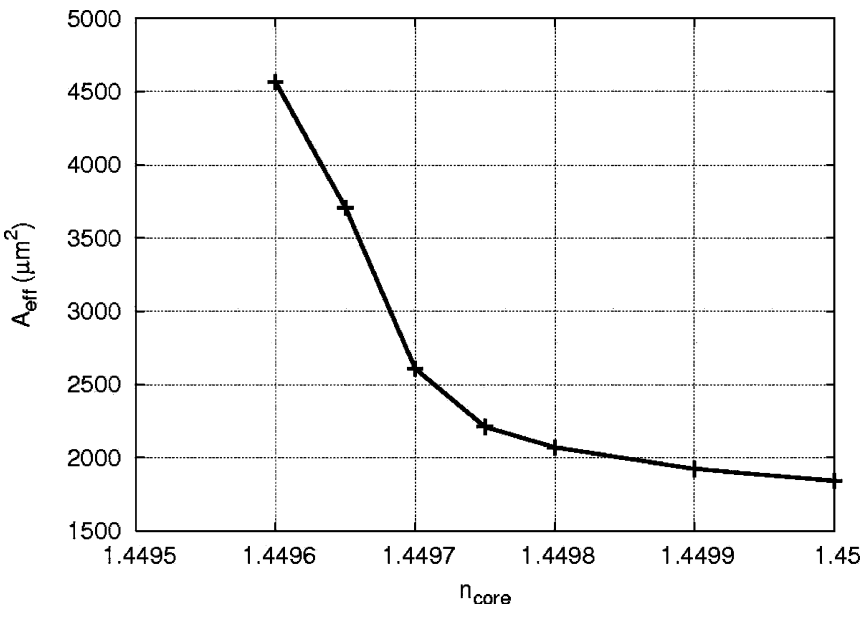

Fig. 5. Effective area of the FM at $1064 \mathrm{~nm}$ versus the core refractive index.

TABLE I

SUMMARY OF THE GEOMETRICAL CHARACTERISTICS OF THE CONSIDERED ROD-TYPE PCFS

\begin{tabular}{|c|c|c|c|c|c|}
\hline $\begin{array}{l}\text { Rod-type PCF } \\
\text { (core diameter) }\end{array}$ & $\begin{array}{c}\Lambda \\
(\mu \mathrm{m})\end{array}$ & $\begin{array}{c}\mathrm{d} \\
(\mu \mathrm{m})\end{array}$ & $\mathrm{d} / \Lambda$ & $\begin{array}{c}\text { holes } \\
\text { ring. }\end{array}$ & $\begin{array}{c}\text { rem. } \\
\text { holes }\end{array}$ \\
\hline $35 \mu \mathrm{m}$ & 12 & 3.96 & 0.33 & 4 & 7 \\
\hline $60 \mu \mathrm{m}$ & 12.5 & 2.375 & 0.19 & 4 & 19 \\
\hline $100 \mu \mathrm{m}$ & 20.8 & 3.958 & 0.19 & 4 & 19 \\
\hline
\end{tabular}




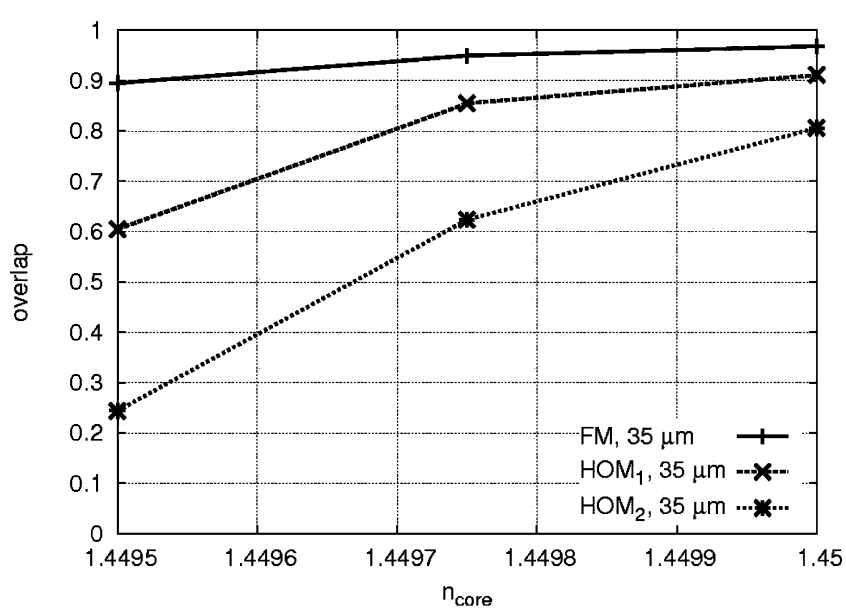

(a)

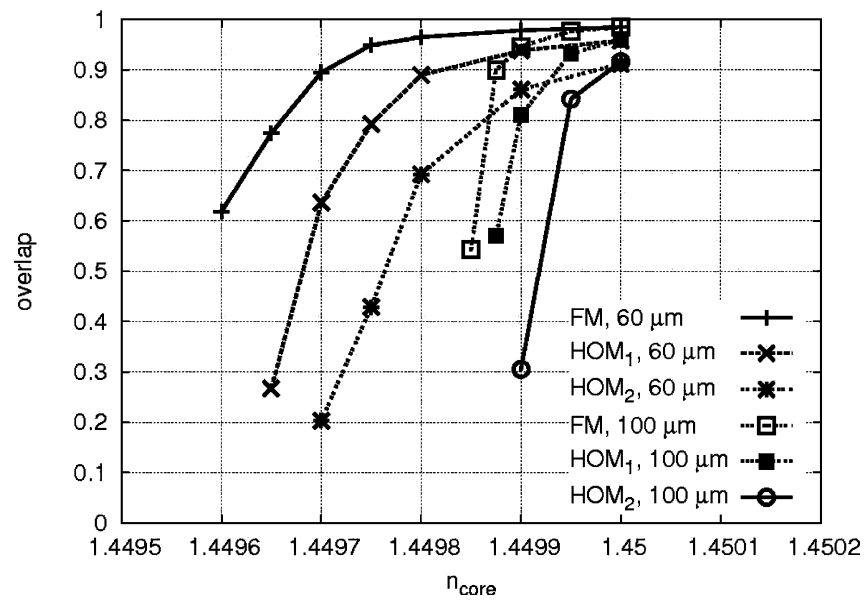

(b)

Fig. 6. Field overlap with the doped core of the FM and HOMs at $1064 \mathrm{~nm}$ versus the core refractive index, for rod-type PCF with core diameters equal to (a) 35 , (b) 60 , and $100 \mu \mathrm{m}$, respectively.

propagation, as will be shown in the next section. The main difference, of course, occurs in the value of the effective area that can be achieved with the three fibers, lower than $950 \mu \mathrm{m}^{2}$ for the $35 \mu \mathrm{m}$ fiber and higher than $2500 \mu \mathrm{m}^{2}$ for the others, at 1064 $\mathrm{nm}$. These results clearly suggest that a proper choice of the doped core refractive index can allow to design rod-type PCFs with very large mode area, in single-mode regime, or with very few HOMs that, however, present a low overlap with the doped core region. It must be noted that such a design will require a very precise control over the core refractive index, and that this accuracy becomes more critical with increasing core size.

\section{AMPLIFIED BEHAVIOR}

In order to verify some conclusions drawn in the previous section while discussing the overlap results, the amplification properties of the rare-earth-doped rod-type PCFs have been investigated considering the mode propagation along the pumped fiber. Still focusing on the $60-\mu \mathrm{m}$ rod-type $\mathrm{Yb}$-doped PCF, a dopant concentration equal to $4.39 \times 10^{25} \mathrm{ions} / \mathrm{m}^{3}$ and a lifetime of the excited state equal to $1 \mathrm{~ms}$ have been considered. The ytterbium absorption and emission cross-sections have been

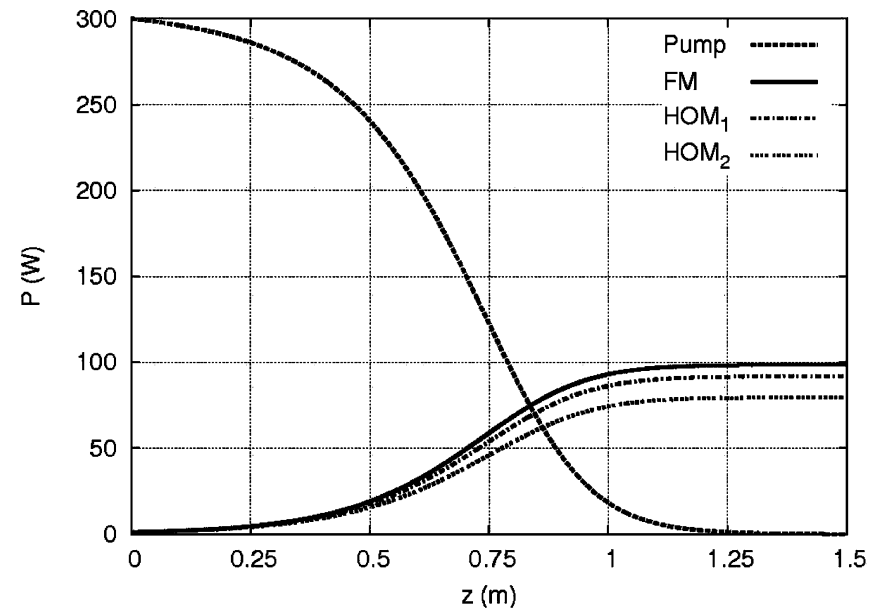

(a)

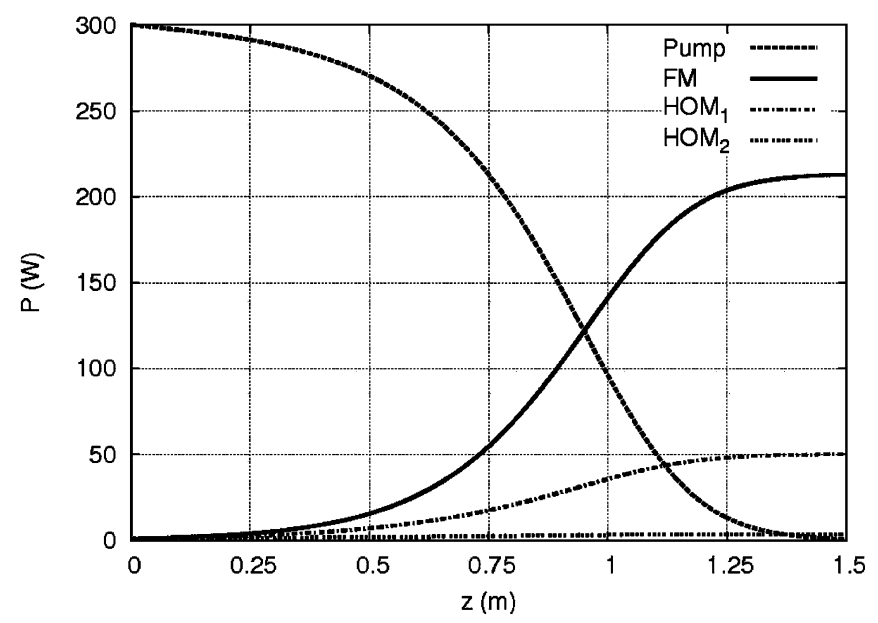

(b)

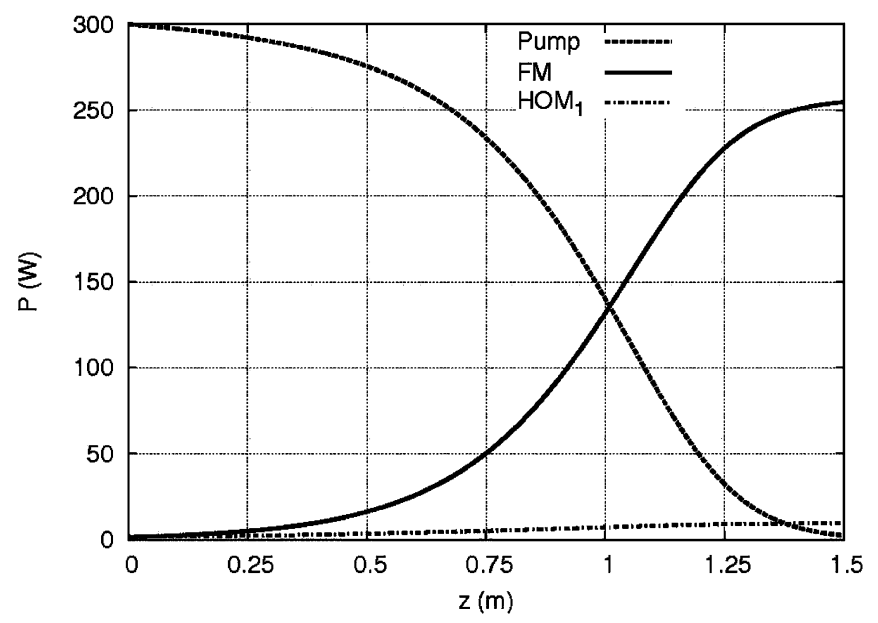

(c)

Fig. 7. Evolution of the power for the pump, FM, and HOMs along the doped rod-type PCF with (a) $n_{\text {core }}=1.45$, (b) $n_{\text {core }}=1.4497$, and (c) $n_{\text {core }}=$ 1.44965 , with a total input power level of $3 \mathrm{~W}$ equally distributed among the modes.

assumed as in [16], and a copropagating pump at $976 \mathrm{~nm}$ with a power of $300 \mathrm{~W}$ has been used for a 1.5-m-long fiber. Results obtained by launching a total input power level of $3 \mathrm{~W}$ equally distributed among the FM and the HOMs are reported in Fig. 7. 


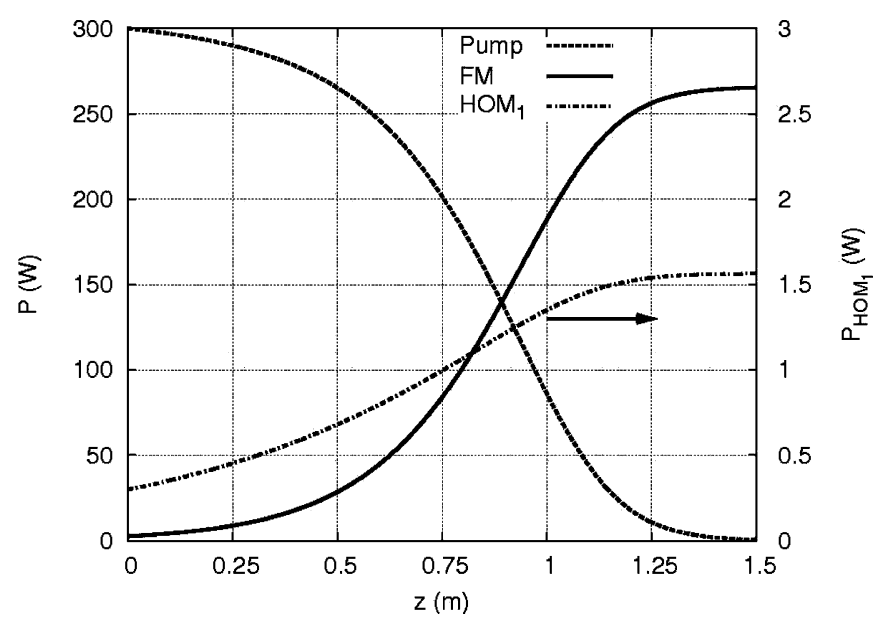

Fig. 8. Evolution of the power for the pump, FM, and $\mathrm{HOM}_{1}$ along the doped rod-type PCF with $n_{\text {core }}=1.44965$. The distribution of $3 \mathrm{~W}$ input power among modes is $90 \%$ to the $\mathrm{FM}$ and $10 \%$ to the $\mathrm{HOM}_{1}$.

Notice that this is a worst-case assumption as actual excitation conditions of the fiber usually favors the FM, rather than equally distributing the input power among all the propagating modes. Fig. 7 shows the different amplification undergone by the modes according to the values of the core refractive index, i.e., as observed in the previous section, according to the different values of the mode overlaps.

In the first case, corresponding to $n_{\text {core }}=1.45$ and shown in Fig. 7(a), all the three modes achieve a high output power level, being able to exploit in a similar way the population inversion in the doped core, as clearly described by the comparable values of the overlap in Fig. 4. When passing to $n_{\text {core }}=1.4497$, the overlaps strongly differ, being about 0.62 for $\mathrm{HOM}_{1}$, and with $\mathrm{HOM}_{2}$ $n_{\text {eff }}$ below $n_{\mathrm{SFM}}$. In these conditions, the mode competition favors the FM that achieves an output power level higher than 200 W, as reported in Fig. 7(b). Finally, with $n_{\text {core }}=1.44965$, both the HOMs are below the $n_{\mathrm{SFM}}$ curve. Only $\mathrm{HOM}_{1}$ is considered for the propagation in the doped fiber, $\mathrm{HOM}_{2}$ being no more guided. Fig. 7(c) shows that the pump depletion is practically given only by the FM, which reaches a $250 \mathrm{~W}$ output power level with a very high conversion efficiency. Note that the two considered modes exhibit the same input power of $1.5 \mathrm{~W}$, but at the output of the doped fiber, the power of $\mathrm{HOM}_{1}$ is only $8 \%$ with respect to the FM mode one.

Fig. 8 reports results obtained for a more realistic case. The distribution of $3 \mathrm{~W}$ input power among modes is $90 \%$ to the FM and $10 \%$ to $\mathrm{HOM}_{1}$. In this case, the output power of $\mathrm{HOM}_{1}$ is only $0.5 \%$ with respect to the FM mode.

\section{CONCLUSION}

The possibility to obtain single-mode or effectively singlemode regime in LMA DC rod-type PCFs has been discussed. It has been numerically demonstrated how a slight core down-doping can push the high-order modes below the SFM effective index curve, or can provide overlap values with the doped core that assure the amplification of the FM to the detriment of the HOMs eventually excited in the fiber.
Examples of rod-type PCFs with 35, 60, and $100 \mu \mathrm{m}$ core diameters have been considered, demonstrating, in all cases, that the doped core refractive index can be engineered to assure high-power single-mode output beams.

\section{REFERENCES}

[1] S. Selleri and F. Poli, "Doped fiber lasers: From telecom to industrial applications," presented at the Int. Conf. Transparent Opt. Netw. (ICTON 2008), Athens, Greece, Jun. 22-26, Invited Paper.

[2] E. Snitzer, H. Po, F. Hakimi, R. Tumminelli, and B. C. McCollum, "Double clad, offset core Nd fiber laser," in Optical Fiber Sensors (OSA Technical Digest Series 2). Washington, DC: Optical Society of America, 1988, Paper PD5.

[3] P. St. J. Russell, "Photonic-crystal fibers," IEEE/OSA J. Lightw. Technol., vol. 24, no. 12, pp. 4729-4749, Dec. 2006.

[4] K. Furusawa, A. Malinowski, J. H. V. Price, T. M. Monro, J. K. Sahu, J. Nilsson, and D. J. Richardson, "Cladding pumped Ytterbium-doped fiber laser with holey inner and outer cladding," Opt. Exp., vol. 9, pp. 714 720, Dec. 2001.

[5] W. J. Wadsworth, R. Percival, G. Bouwmans, J. C. Knight, and P. St. J. Russell, "High power air-clad photonic crystal fibre laser," Opt. Exp., vol. 11, pp. 48-53, Jan. 2003.

[6] K. P. Hansen, C. B. Olausson, J. Broeng, K. Mattson, M. D. Nielsen, T. Nikolajsen, P. M. W. Skovgaard, M. H. Sørensen, M. Denninger, C. Jakobsen, and H. R. Simonsen, "Airclad fiber laser technology," presented at the Photon. West 2008, San Jose, CA, Jan. 19-24, Invited Paper

[7] J. Limpert, T. Schreiber, A. Liem, S. Nolte, H. Zellmer, T. Peschel, V. Guyenot, and A. Tünnermann, "Thermo-optical properties of air-clad photonic crystal fiber laser in high power operation," Opt. Exp., vol. 11, pp. 2982-2990, Nov. 2003.

[8] J. Limpert, N. Deguil-Robin, I. Manek-Honninger, F. Salin, F. Röser, A. Liem, T. Schreiber, S. Nolte, H. Zellmer, A. Tünnermaann, J. Broeng, A. Petersson, and C. Jakobsen, "High-power rod-type photonic crystal fiber laser," Opt. Exp., vol. 13, pp. 1055-1058, Feb. 2005.

[9] J. Limpert, O. Schmidt, J. Rothhardt, F. Röser, T. Schreiber, A. Tünnermann, S. Ermeneux, P. Yvernault, and F. Salin, "Extended single-mode photonic crystal fiber lasers," Opt. Exp., vol. 14, pp. 2715 2720, Apr. 2006.

[10] S. Selleri, A. Cucinotta, M. Foroni, F. Poli, and M. Bottacini, "New design of single-mode large-mode-area photonic crystal fibers," in Proc. Int. Congr. Opt. Optoelectron., vol. 5950. Bellingham, WA: SPIE, 2005, pp. 59500U-1-59500U-11.

[11] B. J. Mangan, J. Arriaga, T. A. Birks, J. C. Knight, and P. St. J. Russell, "Fundamental-mode cutoff in a photonic crystal fiber with a depressedindex core," Opt. Lett., vol. 26, pp. 1469-1471, Oct. 2001.

[12] L. Vincetti, F. Poli, and S. Selleri, "Confinement loss and nonlinearity analysis of air-guiding modified honeycomb photonic bandgap fibers," IEEE Photon. Technol. Lett., vol. 18, no. 3, pp. 508-510, Feb. 2006.

[13] S. Selleri, L. Vincetti, A. Cucinotta, and M. Zoboli, "Complex FEM modal solver of optical waveguides with PML boundary conditions," Opt. Quantum Electron., vol. 33, pp. 359-371, Apr. 2001.

[14] A. Cucinotta, F. Poli, and S. Selleri, "Design of erbium-doped triangular photonic crystal fiber based amplifiers," IEEE Photon. Technol. Lett., vol. 16, no. 9, pp. 2027-2029, Sep. 2004.

[15] A. Cucinotta, F. Poli, S. Selleri, L. Vincetti, and M. Zoboli, "Amplification properties of $\mathrm{Er}^{3+}$ doped photonic crystal fibers," IEEE/OSA J. Lightw. Technol., vol. 21, no. 3, pp. 782-788, Mar. 2003.

[16] R. Paschotta, J. Nilsson, A. C. Tropper, and D. C. Hanna, "Ytterbiumdoped fiber amplifiers," IEEE J. Quantum Electron., vol. 33, no. 7, pp. 1049-1056, Jul. 1997.

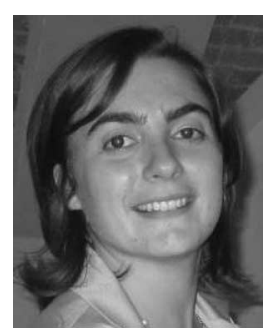

Federica Poli was born in Reggio Emilia, Italy, in 1977. She received the Laurea degree (five years) in electronic engineering and the Ph.D. degree in information technology from the University of Parma, Parma, Italy, in 2002 and 2006, respectively.

She is currently a Postdoctoral Researcher in the Department of Information Engineering, University of Parma. Her current research interests include photonic crystal fibers, optical fiber amplifiers, optical fiber sensors, and integrated optical components. 


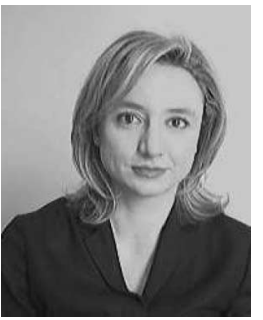

Annamaria Cucinotta (M'08) was born in Parma, Italy, in 1969. She received the Ph.D. degree in information technology from the University of Parma, Parma, Italy, in 1999.

From February 2000 to December 2003, she was a Contract Researcher in the Department of Information Engineering, University of Parma, where, since December 2003, she has been an Assistant Professor with the Faculty of Engineering. Her current research includes fiber laser sources, photonic crystal fibers, electromagnetic field analysis.

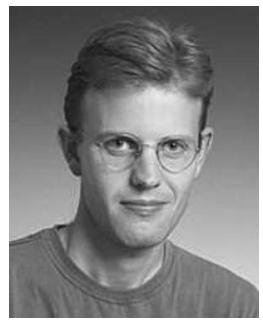

Jesper Lægsgaard was born in Skanderborg, Denmark, in 1970. He received the Ph.D. degree in theoretical physics from the University of Aarhus, Aarhus, Denmark, in 1998.

Since 1998, he has been a Researcher Fellow at the Technical University of Denmark, Copenhagen, Denmark, where he became an Associate Professor in 2004. His current research interests include nonlinear propagation in photonic crystal fibers and fiber lasers utilizing advanced microstructured fibers.

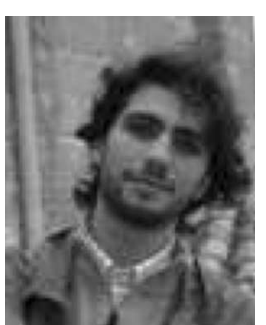

Davide Passaro (S'08) was born in Manduria, Italy, in May 1982. He received the Laurea degree in telecommunication engineering in 2006 from the University of Parma, Parma, Italy, where he is currently working toward the Ph.D. degree in the Department of Information Engineering.

His current research interests include optical fiber amplifiers, photonic crystal fibers, and optical fiber sensors.

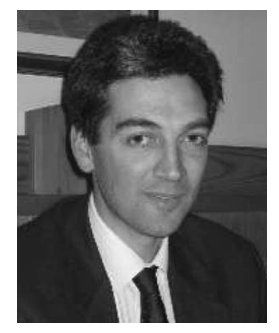

Stefano Selleri (SM'08) was born in Bologna, Italy, in 1966. He received the Laurea degree (cum laude) in electronic engineering from Bologna University, Bologna, in 1991, and the Ph.D. degree from the University of Parma, Parma, Italy, in 1995.

Since 1997, he has been a Researcher in the Department of Information Engineering, University of Parma, where he became an Associate Professor in March 2002. His current research interests include numerical methods for modal and propagation analysis of electromagnetic fields in conventional, photonic crystal, and holey fibers, as well as conventional and photonic crystal integrated optics waveguides, optical amplification, fiber-based amplifiers and lasers, and optical sensing.
Jes Broeng was born in Copenhagen, Denmark, in 1971. He received the Ph.D. degree from the Technical University of Denmark, Copenhagen, in 2000.

$\mathrm{He}$ is currently with Crystal Fibre A/S, Birkerod, Denmark, where he is engaged in fiber research and development.

Dr. Broeng was the Co-Founder of Crystal Fibre A/S. He was the recipient of the 1999 European Optics Prize for his work on photonic bandgap fibers and the 2004 Photonics Circle of Excellence Award for commercialization of active photonic crystal fiber products. 